For $A=\frac{1}{4}$ the crystal consists of regions containing exclusively one or the other kind of layer. If $\varphi=2 \pi n$, equation (2) gives sharp interference maxima in the same positions as the maxima of the ordered scattering and the intensities are proportional to $\left[\left(V_{1}-V_{2}\right) / 2\right]^{2}$. The total intensity of the reflections is proportional to $\left[\left(V_{1}+V_{2}\right) / 2\right]^{2}+\left[\left(V_{1}-V_{2}\right) / 2\right]^{2}$, which is the average intensity of two crystals with the structure amplitudes $V_{1}$ and $V_{2}$. The calculated structure amplitudes for this structure and the random structure are compared with those observed in Table 2.

Since there are two types of layers, $A$ cannot be exactly $\frac{1}{4}$ and some disorder streaking is expected near the reflections where $h-k \neq 3 n$, and the streaking will be more intense near the stronger reflections. For those reflections where $h-k=3 n,\left[\left(V_{1}-V_{2}\right) / 2\right]^{2}$ is zero and no streaking should be observed. That this is the case may be seen in Fig. 1. The streaking between the $(10.4)$ and (10.5) reflections appears to be stronger than one would expect in comparison to the streaking between the other reflections, but this can be explained by considering the boundaries of the regions where a superlattice with a repeat period of eighteen close-packed layers is formed by the two different layers. One would then expect maxima to lie between the regular reflections, but they would be diffuse owing to the fact that the size of diffracting regions is small. The streaking between the $(10.4)$ and (10.5) reflections would correspond to the reflection (10.9) of the larger unit cell, and indeed the calculated intensity of this reflection is five times stronger than any other superlattice reflection.

\section{Discussion}

Samarium is one of the few rare earths with a relatively stable valence of +2 . Whether the stability of such a state is reflected in the metal is a matter of some interest. According to Pauling's theory of valence (1947) the number of valence electrons should materially influence bond distances, so that any contribution to the metal of +2 rather than +3 valence should increase the metal-metal distance and reduce the density of samarium relative to the normal sequence of rare earth metals. As nearly as can be judged there is no such effect, so that the metallic valence of samarium is probably close to three.

It is not known why the structure of samarium deviates from a cubic or hexagonal close-packed arrangement but it is not the only rare earth metal which does so. Klemm \& Bommer (1939) reported evidence (they referred to K. Meisel's work) requiring the $c$ axis of praseodymium and neodymium to be doubled. This has been confirmed for neodymium by Ellinger \& Zachariasen and by us. In this case the packing sequence is $a b c b$.

\section{References}

DaAne, A. H., Dennison, D. H. \& Spedding, F. H. (1953). J.Amer. Chem. Soc. 75, 2272.

Ellinger, F. H. \& Zachariasen, W. H. (1953). J. Amer. Chem. Soc. 75, 5650.

Hendricks, S. B. \& Tellek, E. (1942). J. Chem. Phys. $10,147$.

Klemm,W. \& Bommer, H. (1939). Z. anorg. Chem. 241, 264. Pauling, L. (1947). J. Amer. Chem. Soc. 69, 542.

Acta Cryst. (1954). 7, 535

\title{
The Geometrical Basis of Crystal Ghemistry. Part 1
}

\author{
By A. F. WeLls \\ Imperial Chemical Industries Limited (Dyestuffs Division), Hexagon House, Manchester 9, England
}

(Received 1 January 1954 and in revised form 1 April 1954)

An investigation is made of the periodic three-dimensional systems of points in which each point is connected to three others. These nets form the bases of the structures of a large number of crystals in which there are directed bonds or hydrogen bonds.

\section{Introduction}

The principles underlying the crystal structures of some groups of compounds, for example, ionic, molecular, and the simpler metallic systems, are known at least in broad outline. Less attention seems to have been paid to the large class of crystals whose structures are determined by systems of directed bonds extending indefinitely in one, two, or three dimensions. These include: many non-metallic and 'semi-metallic' elements; oxides of some non-metals; oxides, halides and sulphides of some less electropositive and $B$ subgroup metals; certain borides, carbides and silicides; and some oxy-salts and complex oxides (e.g. silicates). The other major group of crystal structures to be regarded in this way embraces all hydrogen-bonded molecular crystals. The atoms taking part in the hydrogen bonding act, from the structural point of view, like localized charges and result in limited numbers of relatively close intermolecular contacts. 
These include: molecules containing $\mathrm{OH}$ groups (e.g. $\mathrm{H}_{2} \mathrm{O}, \mathrm{H}_{2} \mathrm{O}_{2}$ ), organic hydroxy compounds, oxy-acids, carboxylic acids, molecules containing $\mathrm{CO}$ and $\mathrm{NH}$ groups etc.

If in a crystalline dihydroxy compound each $\mathrm{OH}$ group forms $\mathrm{O}-\mathrm{H}-\mathrm{O}$ bonds to two $\mathrm{OH}$ groups of neighbouring molecules the structural unit may be represented diagrammatically

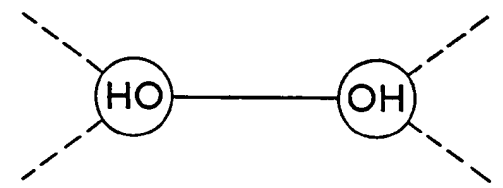

so that the whole crystal may be represented as a 3 -connected network of points ( $\mathrm{OH}$ groups) and lines.

Just as the simplest planar arrangement of atoms forming three equivalent bonds is found in the hexagonal layers of crystalline graphite, so it is to be expected that the three-dimensional analogues of the plane nets will be important as the basic frameworks underlying the crystal structures of many substances, both inorganic and organic. These three-dimensional periodic polygonal frameworks do not appear to have been derived systematically, though their importance in crystallography would seem self-evident. In the simplest case such a framework (net) of linked points represents the structure of an element, each point being an atom, but we shall show that these nets have a much wider application.

An advantage of describing such frameworks as systems of polygons rather than as sets of points generated by groups of symmetry operations is that a number of substances may have essentially the same structure but distorted in different ways so as to have, for example, tetragonal, orthorhombic, or monoclinic symmetry (as for $\mathrm{PtS}, \mathrm{PdO}$ and $\mathrm{CuO}$ ). Provided that the system of linked polygons is the same then all three structures are described as based on the same three-dimensional net.

In this sense these nets have a deeper structural significance than space groups. The particular symmetry exhibited by an arrangement of atoms or molecules is to be regarded as of secondary importance and determined by metrical factors such as relative lengths of bonds and relative values of interbond angles. The practical importance of space groups lies in the fact that the knowledge of the symmetry of a crystal assists in the determination of the structure, though the simpler structures could be-and in some cases were-determined from X-ray data without recourse to space-group theory.

We shall not generally be concerned here with these metrical factors, whence it follows that the value of the geometrical approach as an aid to structure determination is limited. We may indicate the broad structural possibilities for, say, an organic dihydroxy compound, but the exact way in which the molecules of a particular dihydric phenol are linked up will depend on the internal structure of the molecule.

\section{The scope of the present paper}

We shall derive and describe certain groups of periodic three-dimensional networks of linked points which conform to the following rules:

1. Each point is connected to three others (3-connected net).

2. There must be a configuration of each net in which $(a)$ the distance between unconnected points is greater than the distance between any pair of connected points; $(b)$ it is possible to go from any point of the net to any other, starting along any prescribed link, without traversing the same link twice;* $(c)$ all the links from a given point go to different points, i.e. there are no loops (2-gons). Loops in 3-connected nets are trivial since they are equivalent to a pair of points added along a link. The need for including loops in a complete enumeration of the more highly connected nets will be mentioned in a later paper.

A problem closely related to the present one is the open packing of spheres; its relation to the threedimensional nets will be indicated.

\section{Methods of deriving three-dimensional nets}

Any system of connected points which is periodic in three dimensions can be formed by the repetition, at the points of a lattice, of identical units in the same orientation. For a three-dimensional 3-connected net the number of points in the repeat unit must be even, and the minimum number of points in the repeat unit is 4 , since the unit must be connected to six others:

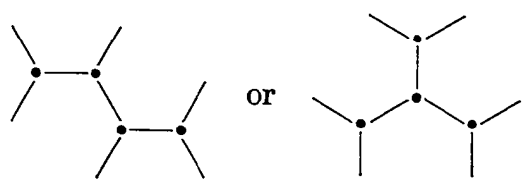

Alternatively three-dimensional nets may be derived by joining up one- or two-dimensional nets containing sufficient $(p-1)$ - or $(p-2)$-connected points to provide the links necessary to form the three-dimensional $p$-connected net. Here we shall derive three-dimensional nets from two-dimensional ones, and for this reason we shall first consider briefly the simpler planar 3 . connected nets.

\section{Two-dimensional nets}

These are derivable by finding the ways in which a plane may be divided into polygons, each point being connected to three others. If $\varphi_{n}$ is the fraction of the total number of polygons which are $n$-gons, then necessarily

* This condition excludes an 'appendix' connected to the main body of the network by a single link. 
<smiles>CC(C)C1C(C)C2C(C)C(C)C3C(C)C(C)C(C)C4C(C(C)C)C(C)C(C1C)C2C34</smiles>

Net $1, N=2$

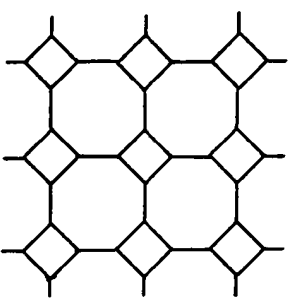

Net $3, N=4$

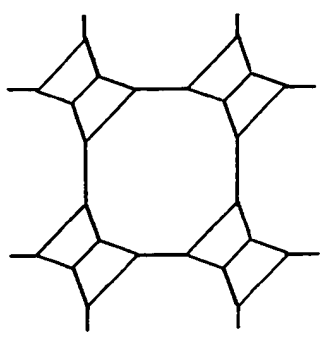

Net $6, N=6$

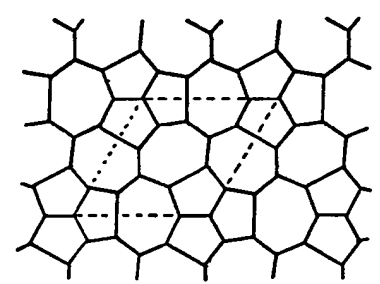

Net $2 a, N=12$

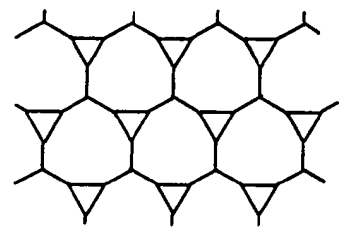

Net $4, N=4$

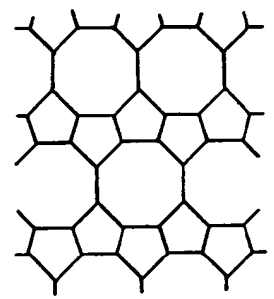

Net $7, N=6$

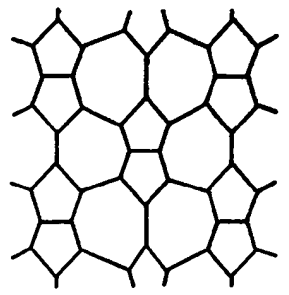

Net $2 b, N=8$

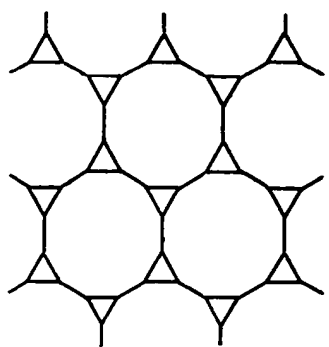

Net $5, N=6$

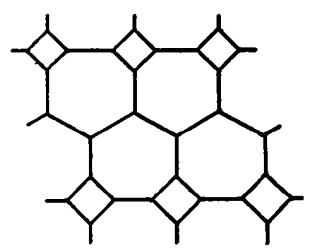

Net $8, N=6$

Fig. 1. Some 3-connected plane nets.

$$
\varphi_{3}+\varphi_{4}+\varphi_{5}+\varphi_{6}+\ldots+\varphi_{n}=1,
$$
and in addition

$$
3 \varphi_{3}+4 \varphi_{4}+5 \varphi_{5}+6 \varphi_{6}+\ldots+n \varphi_{n}=6
$$

\section{Table 1. 3-connected plane nets}

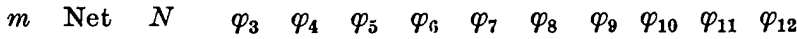

$$
\begin{aligned}
& 1 \quad 1 \quad 2-\ldots-1--\ldots
\end{aligned}
$$

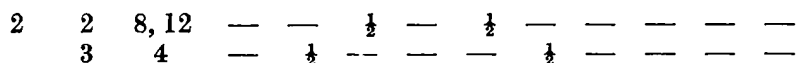

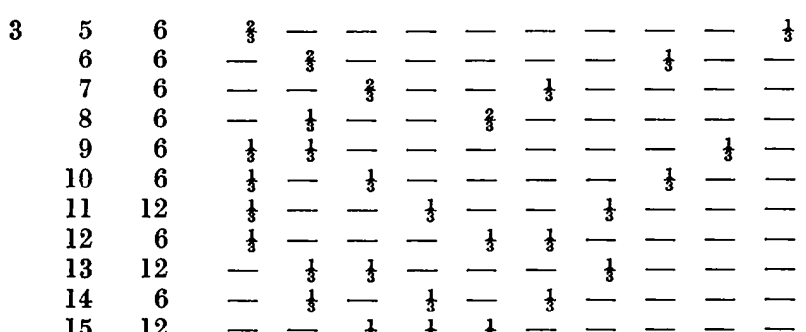
in order of increasing 'order' $m, m$ being the highest denominator in $\varphi_{n}$. For 3-connected nets the number $N$ of points in the repeat unit is $2 m$ or a multiple of $2 m$. For example, there is no configuration of Net 2 with 4 points in the repeat unit, but there are configurations with 8 and 12 points in their repeat units. This illustrates a further point, that specifying the values of $\varphi_{3}$ etc. does not necessarily uniquely define the net, for there may be a number of arrangements of $n_{1}$-gons, $n_{2}$-gons, etc. in the same proportions.

\section{Derivation of three-dimensional 3-connected nets}

These nets may be derived systematically by adding 2-connected points to planar 3-connected nets and then joining each to a 2-connected point of an adjacent layer. It follows that there must be an even number of 2-connected points in the repeat unit. The number of 
points in the repeat units of planar 3-connected nets are 2,4 , etc. (Table 1 ), so that for the three-dimensional 3-connected nets we have the following possibilities:

\section{4 points in repeat unit: 2 2-connected, 2 3-connected}

There is only one planar 3-connected net with 2 points in the repeat unit, giving the nets of Fig. 2.
Net 1

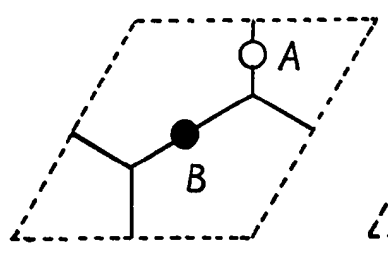

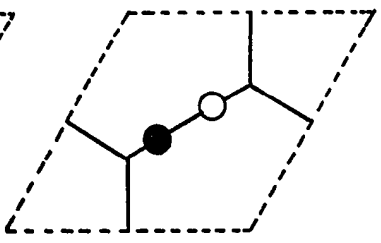

Net 2

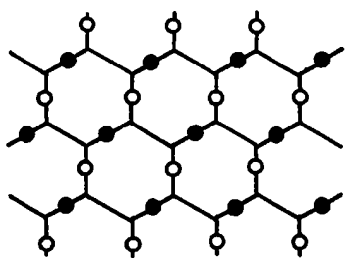

(1)

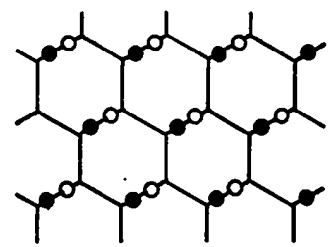

(2)
Fig. 2. Derivation of 3-connected Nets 1 and 2. 0 : point connected upwards $(U)$ to point of layer above; - : point connected downwards $(D)$ to 0 point of layer below.

One of the 2-connected points $(A)$ is to be connected to $B^{\prime}$ of the layer above, the other $(B)$ to $A^{\prime \prime}$ of the layer below. The nets can be more easily visualized if we show a larger portion of the planar net, as indicated in the figure. Net 1 can adopt the highly symmetrical cubic form shown in Figs. 6 and 7, the points being the equivalent points $8(a)$ of the spacegroup $I 4_{1} 3$. This (enantiomorphic) net may be regarded as the 3-connected analogue of the (4-connected) diamond net.

\section{6 points in repeat unit}

The possibilities here are:

(1) Planar net with 2 points in repeat unit+4 2-connected points, considering then the various permutations of $2 U$ and $2 D$ among the 2 -connected points. ( $U$ stands for a point to be connected upwards to a point of the layer above and $D$ for a point to be joined to one of the layer below.)

(2) Planar nets with 4 points in the repeat unit +2 2 -connected points. Here we have to consider the following nets of Table 1: Net 1 (two unit cells), Net 3, and Net 4 . (There is no configuration of Net 2 with only 4 points in the repeat unit.)

It is found that the nets arising from (1) are in- cluded in those derived from the double repeat unit of Net 1, so that we need list only those nets arising under (2). These are set out in Fig. 3, where each

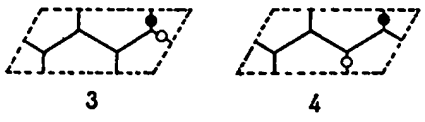

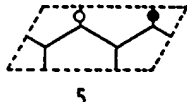

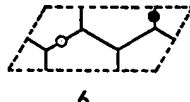

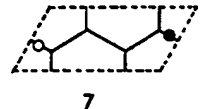

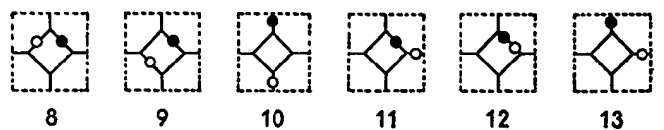

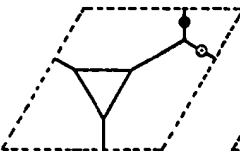

14

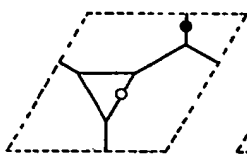

17

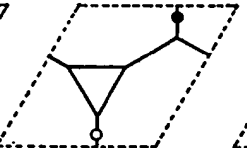

.15

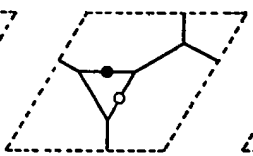

18

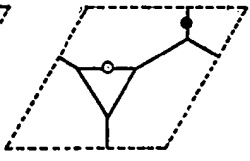

16

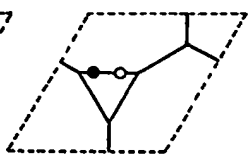

19
Fig. 3. Repeat units in three-dimensional 3-connected nets. The numbers correspond to the numbers of the nets in Table 2.

diagram shows a repeat unit containing 4 3-connected and 2 2-connected points.

This is not a complete enumeration of all 6-point

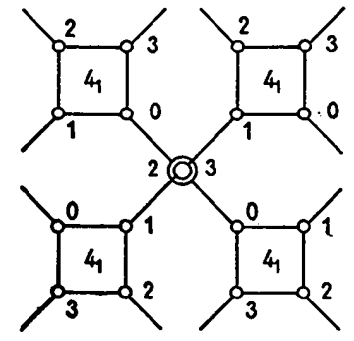

Net 21

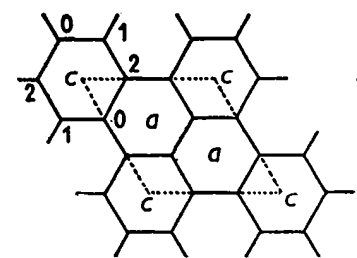

(b)

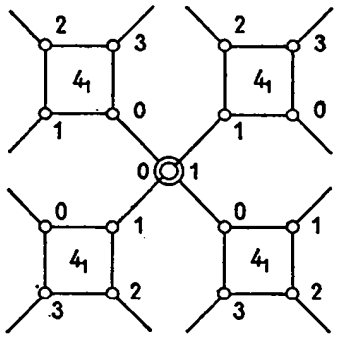

(a)

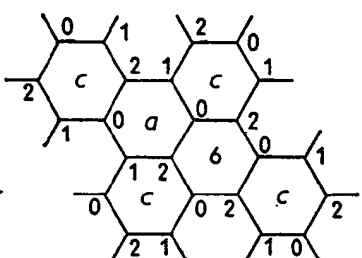

(C)
Fig. 4. (a) Derivation of Nets 20 and 21. (b) Projection of Net 21. (c) Projection of two interpenetrating Nets 9. 
nets. For 3-connected nets having 6 or more points in the repeat unit, nets of the kind shown in Fig. 4(a) arise. In these projections the squares represent clockwise 4-fold helices and the central pairs of points are joined by lines perpendicular to the plane of the paper. The numbers represent heights in terms of onequarter of the unit translation along the screw axis.

Net 21 is a particularly interesting net which in its most symmetrical configuration possesses trigonal symmetry and consists of series of hexagonal helices. If a 6-fold helix is set up at each corner of a hexagonal unit cell and a second helix starts $d / 2$ out of phase with the first ( $d$ being the repeat distance along a helix) then the helices may be joined up as shown in Fig. 13, so that all the points form one continuous network. This net is very closely related to the rhombohedral form of Net 9, for if we start with the same system of two 6-fold helices around each vertical axis but join up as shown in (c) of Fig. 4 then this projection represents, not a single Net 9 , but two identical nets completely interpenetrating but not connected one to the other. In Fig. $4(b)$ and $(c) c$ and $a$ represent clockwise and anticlockwise helices and 6 a closed hexagon, and the numbers indicate heights in terms of $d / 6$. The smallest polygon in Net 21 is a 12-gon, and this net is one of a special set which we shall discuss later (see 'Uniform nets').

The three-dimensional 3-connected nets are listed in Table 2, where some information is given about the polygons of which they are built.

\section{Table 2. Three-dimensional 3-connected nets}

\section{Polygons in net}

\begin{tabular}{|c|c|c|c|c|c|c|c|c|c|c|c|c|c|}
\hline Net & $N$ & 3 & 4 & 5 & 6 & 7 & 8 & 9 & 10 & 11 & 12 & 13 & 14 \\
\hline 1 & 4 & - & - & $\longrightarrow$ & - & - & - & - & 10 & - & - & - & - \\
\hline 2 & & - & - & - & - & - & - & - & 10 & - & - & $\longrightarrow$ & 一 \\
\hline 3 & 6 & - & - & - & - & 7 & - & 9 & 10 & - & - & - & 14 \\
\hline 4 & & - & - & - & - & - & 8 & - & 10 & - & - & - & - \\
\hline 5 & & - & $\longrightarrow$ & - & $\longrightarrow$ & $\longrightarrow$ & 8 & - & - & - & 12 & - & - \\
\hline 6 & & - & - & - & - & - & 8 & - & - & - & - & - & 14 \\
\hline 7 & & - & - & 一 & 6 & - & - & - & 10 & 一 & 12 & - & 14 \\
\hline 8 & & - & - & - & 6 & - & - & - & 10 & - & 12 & - & 14 \\
\hline 9 & & - & - & - & 6 & - & 一 & - & 10 & - & - & $\longrightarrow$ & $\longrightarrow$ \\
\hline 10 & & $\longrightarrow$ & 4 & $=$ & - & - & 一 & - & - & $\overline{7}$ & 12 & - & 14 \\
\hline 11 & & - & - & 5 & 一 & - & - & - & - & 11 & - & - & - \\
\hline 12 & & - & - & - & 6 & - & - & - & 10 & - & 12 & - & 14 \\
\hline 13 & & - & 4 & - & $\longrightarrow$ & - & 8 & - & 一 & - & - & - & 14 \\
\hline 14 & & 3 & - & - & - & - & - & - & - & - & 12 & 13 & - \\
\hline 15 & & 3 & - & $\longrightarrow$ & 一 & - & - & - & - & - & 12 & 13 & 14 \\
\hline 16 & & - & 4 & - & - & - & - & - & - & - & 12 & 一 & 14 \\
\hline 17 & & - & 4 & - & - & 一 & - & - & - & $\overline{-}$ & 12 & - & 14 \\
\hline 18 & & - & - & 5 & - & - & - & - & - & 11 & 12 & - & 14 \\
\hline 19 & & - & - & 5 & - & - & - & - & - & 11 & 12 & 13 & 14 \\
\hline 20 & & - & - & - & - & - & 8 & - & 10 & - & 12 & - & - \\
\hline 21 & & - & - & - & 一 & - & - & $\longrightarrow$ & $\Gamma$ & $\rightarrow$ & 12 & $\rightarrow$ & 14 \\
\hline
\end{tabular}

Certain nets can adopt configurations with rather symmetrical arrangements of links at each point, for example:

3 coplanar bonds at $120^{\circ}$ : Nets $1,2,5,6,7$;

3 coplanar bonds (angles, $\left.150^{\circ}(2), 60^{\circ}\right)$ : Nets 14, 15;

3 coplanar bonds (angles, $\left.135^{\circ}(2), 90^{\circ}\right)$ : Net 10.
The following remarks concern particular nets. Net 9 can adopt a configuration with rhombohedral symmetry which can alternatively be derived by placing plane hexagons at the points of a simple cubic lattice with their planes perpendicular to a body-diagonal of a cubic cell (Figs. $5(b)$ and $6(d)$ ). Net 14 can be derived from Net 1 by replacing one-quarter of the points by triangles (Fig. 5(c) and (d)); Net 15 is related in exactly

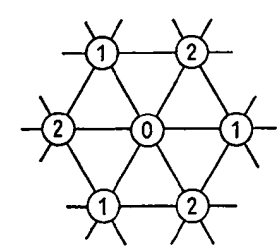

(a)

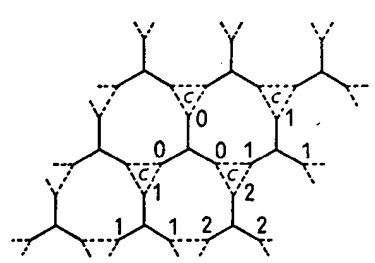

(c)

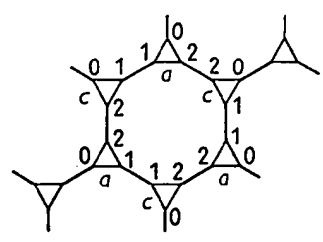

(e)

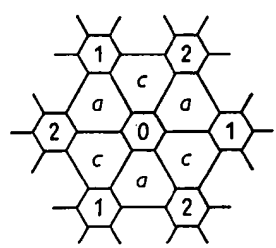

(b)

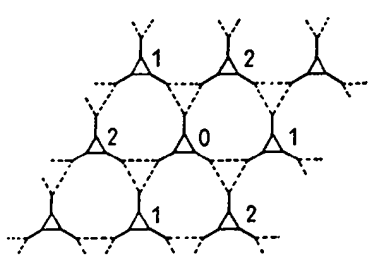

(d)

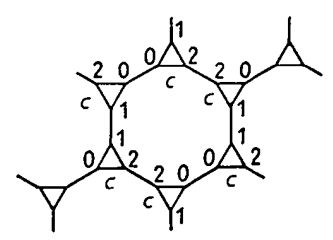

(f)
Fig. 5, (a) Cubic lattice viewed along [111]. (b) Net 9 viewed along a 3-fold axis. $c$ and $a$ indicate clockwise and anticlockwise helices. (c) Net 1 viewed along 3 or $3_{1}$ axis. (d) Net 14, similar projection. (e) Projection of Net 5 . (f) Projection of Net 6. (Numbers indicate heights in terms of one-third of the unit translation perpendicular to plane of paper.)

the same way to Net 2 . For another projection of Net 1 , along a $4_{1}$ axis, see Fig. $14(b)$.

Nets 5 and 6 are closely related. Both can be constructed with three equal coplanar bonds from each point inclined at $120^{\circ}$ and both project as the same two-dimensional net (Fig. $5(e)$ and $(f)$ ). The triangles in the projection of Net 5 represent $3_{1}$ and $3_{2}$ axes, but in the projection of Net 6 they represent all $3_{1}$ (or $3_{2}$ ) axes. In the projection of Net 5 the 12-gons represent closed 12-gons; in the projection of Net 6, helices. Both structures are very open, having tunnels through them.

Nets $1,2,7,9$ and 10 of Table 2 are illustrated in Fig. 6. In addition, Nets $1,2,5,6,9,10$, and 21 are illustrated by pairs of stereoscopic photographs in Figs. 7-13.

No attempt has been made to derive systematically 


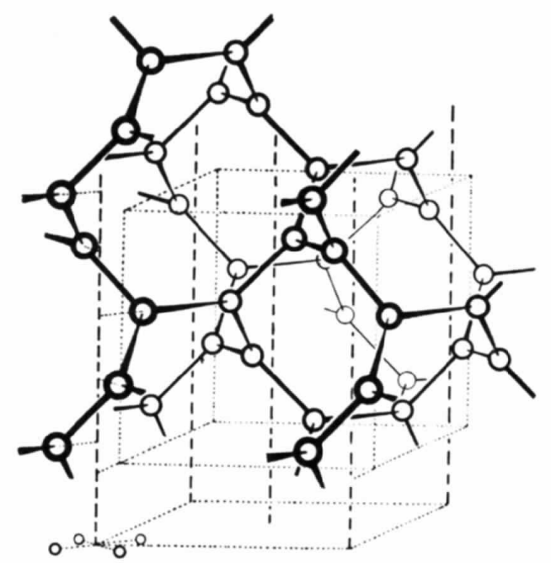

(a)

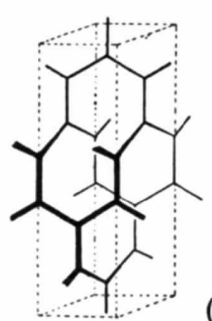

(b)

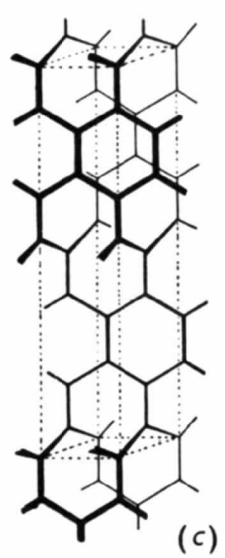

(c)
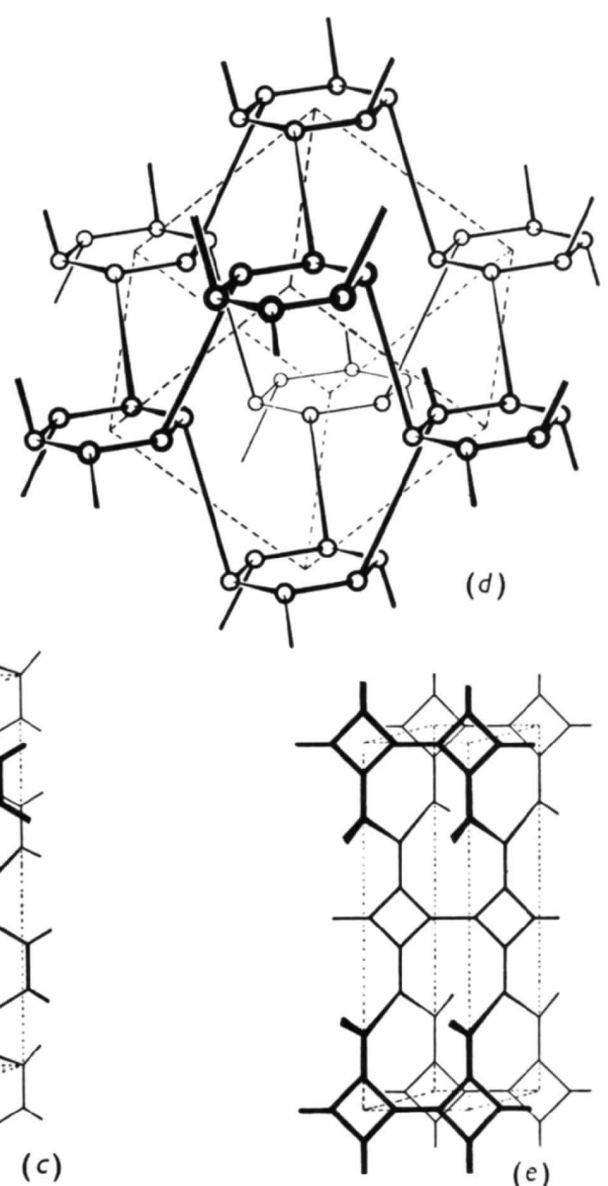

(e)

Fig. 6. Three-dimensional 3-connected nets. (a) Net 1. (b) Net 2. (c) Net 7. (d) Net 9. (e) Net 10.

nets with more than 6 points in the repeat unit. However, the nets of Table 2 suggest families of more complex nets, some of which are likely to be important in crystal chemistry.

\section{Some families of more complex nets}

The projections of three-dimensional nets on suitable planes are simple plane nets, as illustrated for Net 21 in Fig. $4(b)$ and for Net 9 in Fig. $5(b)$. The polygons in the plane net represent either closed polygons or helical arrays of points in the three-dimensional net of which it is one projection. This suggests an alternative way of deriving families of three-dimensional nets all of which project as the same plane net. In the plane net of Fig. 14(a) some or all of the polygons may represent the projections of points generated by screwaxes $\left(4_{1}\right.$ or $4_{3}, 8_{1}$ or $\left.8_{7}\right)$ so that we can visualize a family of 3-connected nets all having this plane net as one projection. Starting with the nets having 4 points in the repeat unit we have first Fig. 14(b), which is the projection of Net 1 of Table 2. For 8 points in the repeat unit there are the possibilities $(c)-(h)$, of which $(e)$ and $(f)$ are illustrated in Fig. 15
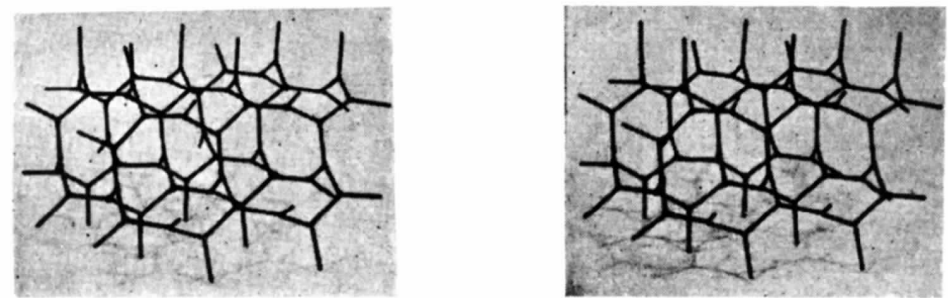

Fig. 7. Net 1. 


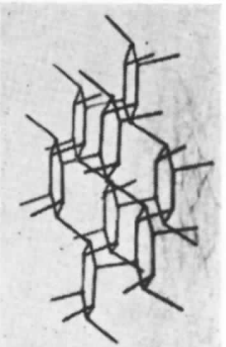

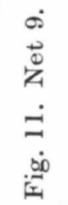
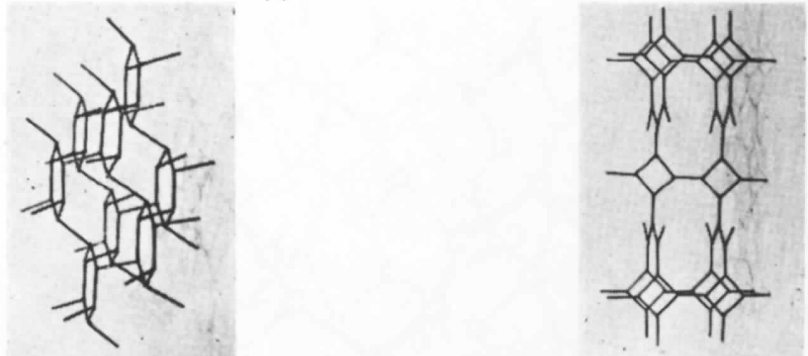

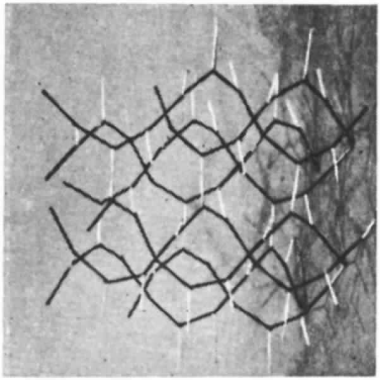

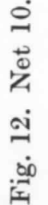

守
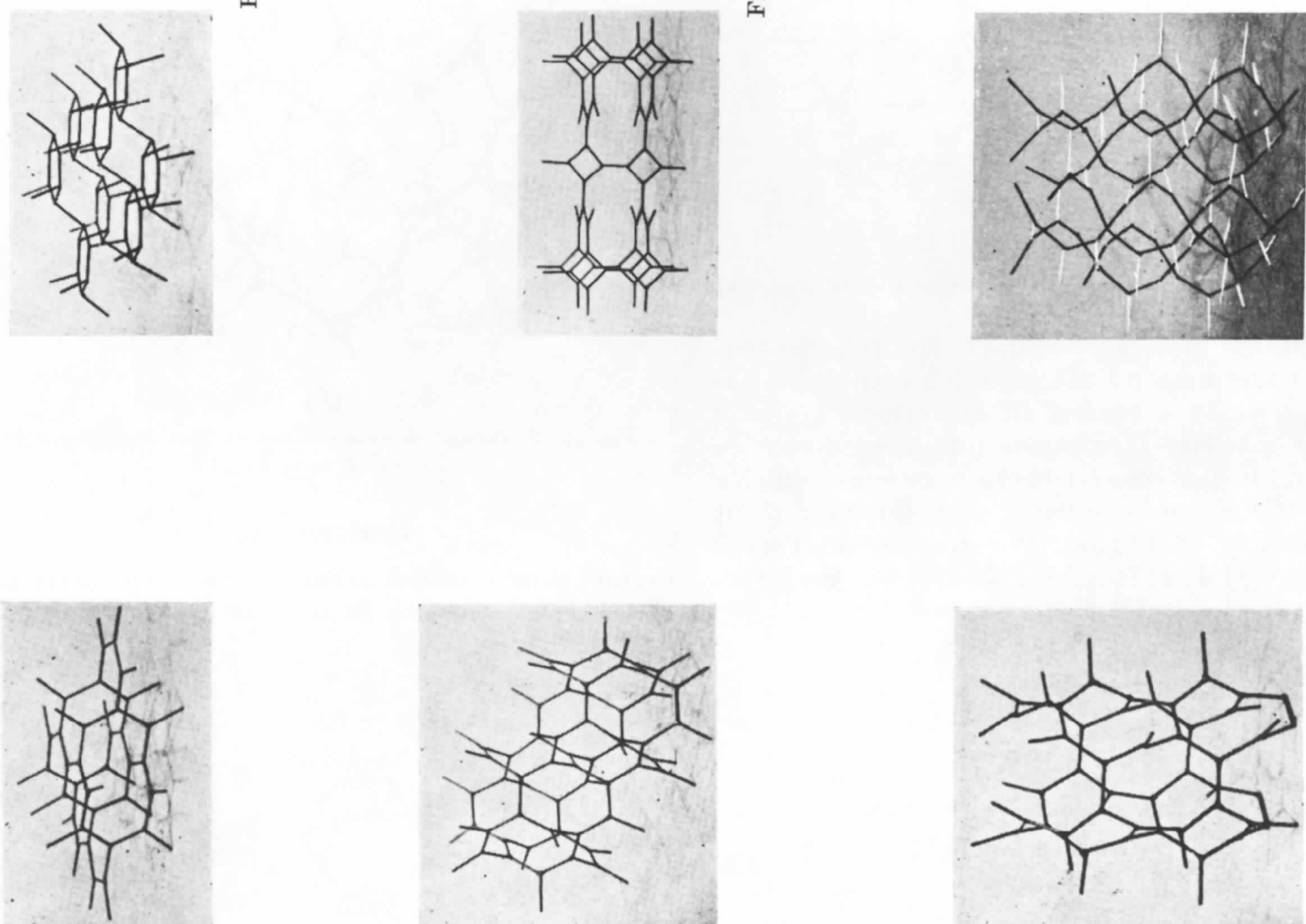

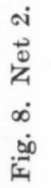
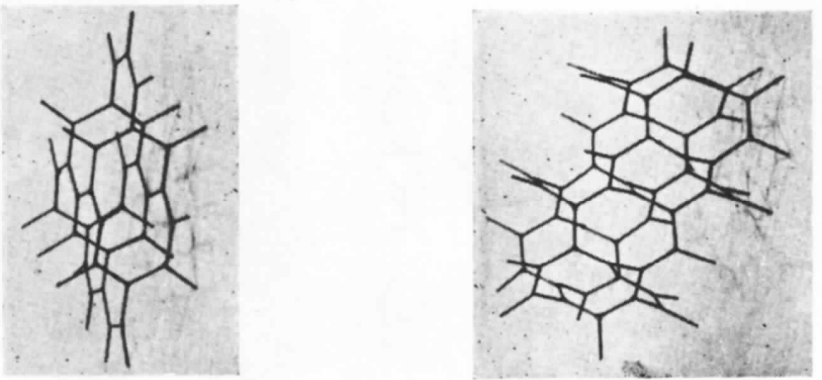

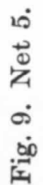

0
$\dot{0}$
$\dot{0}$
$\dot{0}$
$\dot{0}$

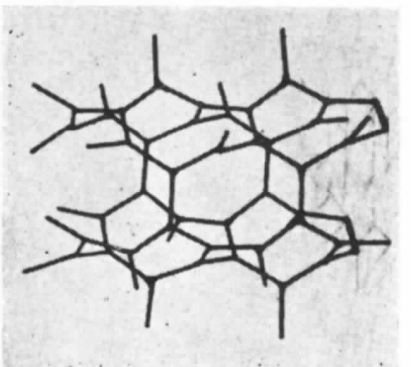




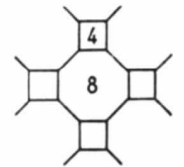

(a)

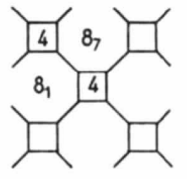

(c)

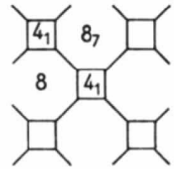

(d)

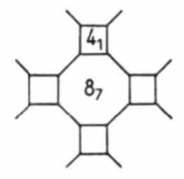

(b)

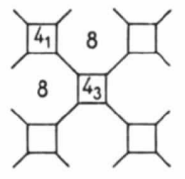

(e)

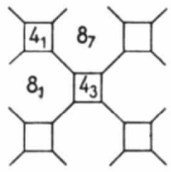

(f)

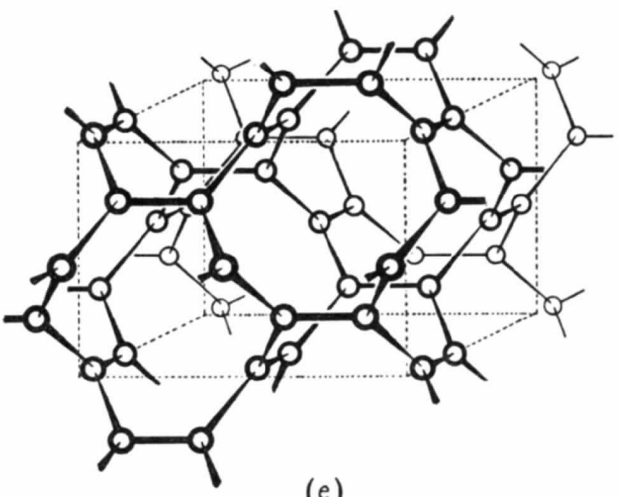

(e)

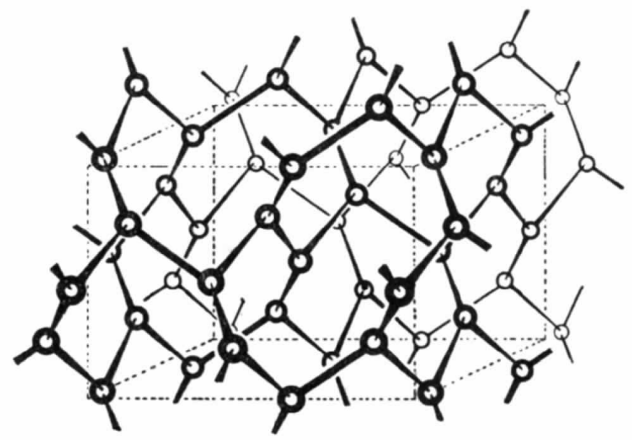

$(f)$

and $(c)$ and $(g)$ in Figs. 16 and 17. The net $(c)$ corresponds to the diamond net in which each point has been replaced by a square. Of the others, $(f)$ is of particular interest. It contains no closed 4-gons or 8-gons, and in this sens' it is the next more complex member of the family starting with $(b)$. Like $(b)$ it is an assembly of 10-gons. We shall see in Part 3 that $(b)$ and $(f)$ do in fact form the bases of the structures of two closely related crystals.
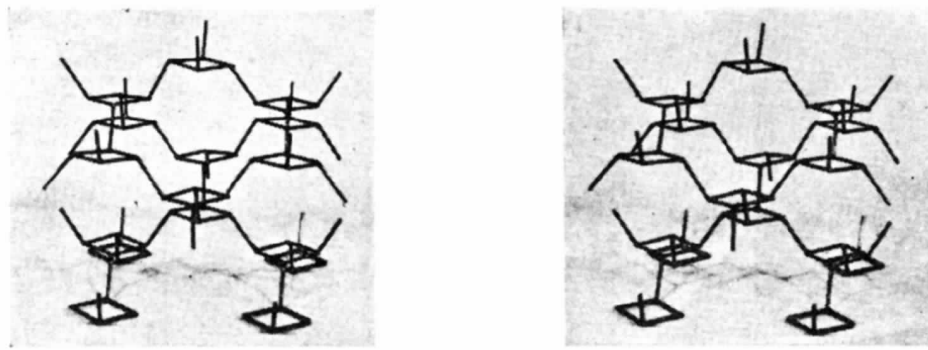

Fig. 16. The net shown in plan in Fig. 14(c).
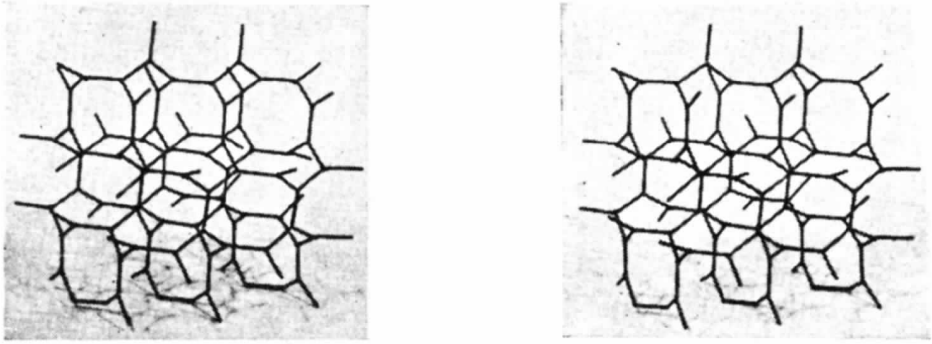

Fig. 17. The net shown in plan in Fig. 14(g). 
Table 3. Uniform three-dimensional 3-connected nets

$\begin{array}{rccccccc}\text { Net } & N & \overbrace{B C(6)} & C D(12) & D E(24) & \text { Symbol } & \text { Space group } & \begin{array}{c}\text { Circuits back to } A \\ \text { Equivalent } \\ \text { position }\end{array} \\ 1 & 4 & 6 & 12 & 18 & 10^{3} & I 4_{1} 3 & 8(a) \\ 2 & 4 & 6 & 10 & - & 10^{3} & I 4 / a m d & 8(e) \\ 5 & 6 & 6 & 8 & 8 & 8^{3} & R \overline{3} m & 18(f)^{*} \\ 6 & 6 & 6 & 8 & 8 & 8^{3} & C 6_{2}^{2} & 6(i)^{*} \\ 21 & 6 & 4 & 4 & - & 12^{2} .14 & C 6_{2}^{2} & 6(g)^{*}\end{array}$

* In the Nets 5, 6 and 21 a variable parameter $x$ must lie within certain limits and also there must be an appropriate value of the other variable $c / a$ in order that no neighbours in excess. of three come closer to a point than the three to which it is connected. For example, for Net 21 the condition for 3 (and only 3 ) equidistant nearest neighbours is that values of $c / a$ and $x$ correspond to points on the plot of $c / a=9\left(1-4 x+3 x^{2}\right)$ between the intersections with the lines $x=1$ and $c / a=1-2 x$.

along any link and returning (along another link) to the starting point passes through the same total number of links (points). This can alternatively be stated in the form:

Every link belongs to an $n$-gon (shortest circuit). A net satisfying this condition may be called a uniform net.

Of the plane 3-connected nets only the hexagonal Net 1 of Fig. 1 satisfies this condition. Of the threedimensional 3-connected nets five of those listed in Table 2, namely Nets $1,2,5,6$ and 21 are uniform, and so also are those of Fig. $14(f)$ and $(g)$, with respectively 10-gon and 8-gon circuits. In addition, one uniform 9-gon net has been found; its derivation is indicated in Fig. 18 and it is illustrated in Fig. 19.

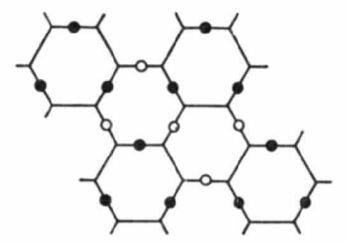

Fig. 18. Derivation of a uniform 9-gon net; compare with Fig. 2.

We may proceed a stage further in classifying uniform nets by considering the choice of paths back to the starting point $A$ (by the minimum $n$-gon circuit) from points $B, C$, etc. removed 1,2 , etc. points from $A$. For a 3-connected net there are six possible paths back to $A$ from the three points $B$ via the six links $B C$,
12 from points $C$ via links $C D$, and so on, and we determine by how many of the $6 \mathrm{BC}, 12 \mathrm{CD}$, etc. paths we can still return to $A$ in an $n$-gon circuit. In this way we see (Table 3 ) that the 3 -connected Net 1 occupies a special position among three-dimensional 3-connected nets. In a similar way it is found that of the 4-connected nets the diamond net (6-gon circuits) has this special property, and of 6-connected nets, the $P$ lattice (4-gon circuits). Corresponding to the three plane nets in which all the polygons are of the same kind:

$\begin{array}{cccc}\text { Two-dimensional } & \text { 3-connected } & \text { 4-connected } & \text { 6-connecte } \\ \text { nets } & \text { 6-gon } & \text { 4-gon } & \text { 3-gon }\end{array}$

we have the special 'most uniform' three-dimensional nets:

Three-dimensional nets 10-gon 6-gon 4-gon

It will be apparent that the above condition for uniformity is not a very restrictive one, requiring only that two $n$-gons meet at each 3 -connected point. Adopting a nomenclature analogous to that for polyhedra, in which the symbol shows the polygons meeting at a point, we find that Net 21 differs from Nets $1,2,5$ and 6 in the same way that the Archimedean solids $m \cdot n^{2}$ differ from the regular solids $n^{3}$ (see Table 3). It would seem, though this point has not been proved, that the only nets of the $n^{3}$ type are $8^{3}$, $9^{3}$ and $10^{3}$, and that there are two different nets corresponding to the symbol $8^{3}$, and similarly for $10^{3}$.
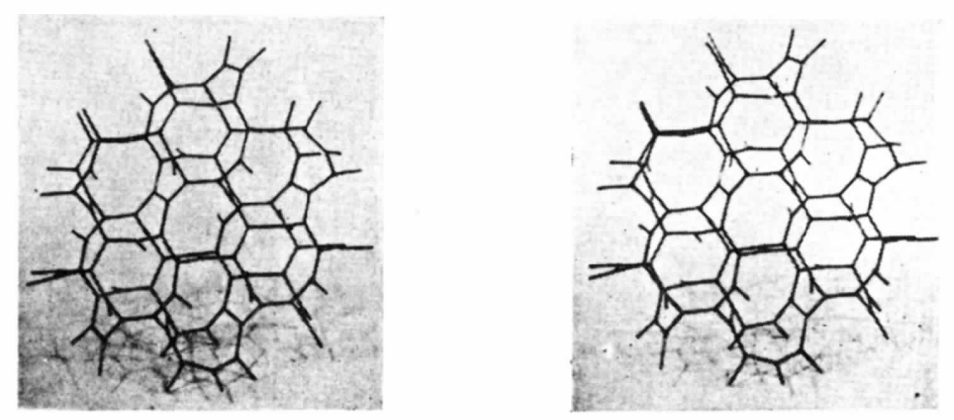

Fig. 19. The uniform 9-gon net of Fig. 18 viewed in a direction nearly parallel to the trigonal axis. 
Table 4. Densities of open packings of equal spheres

Space group
$I 4_{1} 3$
$P 43 m$
$I m 3 m$
$I m 3 m$
$I 4_{1} 3$

Equivalent points

$8(a)$

$24(j): x=\frac{5}{12}, y=\frac{8}{12}, z=\frac{1}{12}$

$48(j): y=\frac{1}{4}, z=\frac{8}{8}$

$24(g): x=0 \cdot 147$

$24(h): x=0.058$
Density
$0 \cdot 185$
$0 \cdot 165$
$0 \cdot 139$
$0 \cdot 117$

$0 \cdot 056$

\author{
Notes \\ 3-connected Net 1. H. \& L. $3_{1}$ \\ H. \& L. $3_{2}$
}

These nets $8^{3}, 9^{3}$ and $10^{3}$ continue the series starting with the 3-connected regular solids, $3^{3}, 4^{3}$ and $5^{3}$, and the planar net $6^{3}$. Similarly, if we replace the points in these three-dimensional nets by triangles then $n^{3}$ becomes $3 . n^{2}$, that is, these derived nets are the analogues of certain of the Archimedean semi-regular solids, the truncated tetrahedron, cube, and dodecahedron, $3.6^{2}, 3.8^{2}$, and $3.10^{2}$. The next member of the series is the plane net, $3.12^{2}$, and from the threedimensional 3-connected nets $8^{3}, 9^{3}$ and $10^{3}$ are derived the nets $3.16^{2}, 3.18^{2}$ and $3.20^{2}$.

Certain of the uniform nets can adopt a configuration with high symmetry in which all the points are equivalent, i.e. the net can be formed by connecting up in the appropriate way the points of one set of equivalent positions in a space-group. This information is included in Table 3 for five of the uniform nets.

\section{Open packings of spheres}

The most open packings of equal spheres for 3- and 4coordination were derived by Heesch \& Laves (1933), who required all the spheres to be crystallographically equivalent and to form one connected, infinite threedimensional system. (Examples of more open packings of non-equivalent spheres were given by Melmore (1942a, $b, 1949)$.) The nets formed by connecting the centres of adjacent spheres are therefore examples of uniform nets of the kind listed in Table 3. Heesch \& Laves were interested in the most open packings and they considered only the most symmetrical structures with cubic or hexagonal symmetry.

The open packings were called:

3-coordination: types $3_{1}$ and $3_{2}$;

4-coordination: types $4_{1}, 4_{2}, 4_{3}$ and $\mathbf{4}_{4}$.
The symbols refer to the topological type, and there may be more than one packing corresponding to a particular symbol.

\section{Type $3_{1}$}

The cubic form is the cubic configuration of the 3 -connected Net 1 (Fig. 7). The rhombohedral and hexagonal variants are the Nets 5 and 6 , illustrated in Figs. 9 and 10.

\section{Type $\mathbf{3}_{2}$}

This arises from the cubic configuration of $3_{1}$ by replacing each point by an equilateral triangle. It is the net $3.20^{2}$ to which we have referred, and it will be illustrated in a later paper.

Heesch \& Laves's packing $\mathbf{3}_{\mathbf{2}}$ is apparently the least dense packing of equal equivalent spheres (though this has never been proved) but there are a number of packings with densities between those of their packings $3_{1}$ and $3_{2}$ of which three examples are given in Table 4 . (The density of a packing is the fraction of the whole space which is occupied by spheres.) The three 24-point nets of Table 4 will be illustrated in a later paper.

Reference will be made to the open packings $4_{1}-4_{4}$ in Part 2, which will be concerned with 4-connected nets.

\section{References}

Heesch, H. \& Laves, F. (1933). Z. Kristallogr. 85, 443. Mecmore, S. (1942a). Nature, Lond. 149, 412.

MelmoRe, S. (1942b). Nature, Lond. 149, 669.

Melmone, S. (1949). Miner. Mag. 28, 479. 